\title{
Finite time non-fragile dissipative control for uncertain TS fuzzy system with time-varying delay
}

\author{
Yuechao Ma, Menghua Chen* \\ ${ }^{a}$ College of Science, Yanshan University, Qinhuangdao Hebei Province, P.R.China, 066004
}

\begin{abstract}
In this paper, the problem of finite time non-fragile dissipative control for uncertain TS fuzzy time-varying delay system is investigated. Considering the time-varying delay and using of a new lemma, delay-dependent conditions are obtained. The closed-loop system is not only finite-time bounded but also satisfies the dissipative control index for all uncertainties under the obtained conditions. Then, the desired non-fragile dissipative controller can be obtained by solving the linear matrix inequalities (LMIs). Finally, simulation results demonstrate the feasibility of the proposed method.
\end{abstract}

Key words: finite time, non-fragile, dissipative, TS fuzzy system, time-varying delay

\section{Introduction}

Over the past two decades, due to their industrial applications, T-S fuzzy systems have been studied extensively. And a great number of significant results on analysis and synthesis problems of T-S systems can be available [1-5]. Uncertain TS fuzzy model was discussed in [6,7]. It has been shown that the existence of time-delays is often one of the main causes of instability and poor performance in a system. Therefore, it has received considerable attention on TS fuzzy time-delay system $[2,6-8]$. On the other hand, since perturbations often appear in the controller gain, which may result from either the actuator degradations or the requirements for readjustment of controller gains. The non-fragile control concept is how to design a feedback control that will be insensitive to some error in gains of feedback control [9-14]. Non-fragile robust $H_{\infty}[10]$ and non-fragile passive controller [11] were discussed. However, the uncertain parameter in $B_{i}$ was ignored in [10], [11] and many other articles on non-fragile control. In practice, it is necessary to consider the uncertainty.

Compared with passivity and $H_{\infty}$ performance, dissipative is a more general criterion, which was introduced by Willems in [15]. However, for nonlinear control system, the design of an efficient dissipative controller is not an easy case. Using the TS fuzzy model, the dissipative design of the nonlinear system is effective and feasible. Hence, the problem of fuzzy dissipative control is significant. H. Gassara et al. obtained

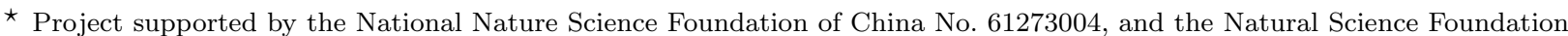
of Hebei province No. F2014203085.

* Corresponding author.

Email address: chenmodehao_220@126.com; telnumber: 86-18637031605; faxnumber: 86-03358057027 (Menghua Chen).
}

Preprint submitted to 
the observer based $(Q, V, R)-\alpha$-dissipative control for T-S fuzzy descriptor systems with time delay in [16]. In [17] and [18], under dissipative conditions, the problem of fuzzy differential equations were discussed. Global robust dissipativity analysis for T-S fuzzy neural networks was demonstrated via LMI approach by Muralisankar et al. [19]. In [20], the problem of fuzzy dissipative control for nonlinear Markovian jump systems via retarded feedback was investigated.

In the aforementioned references, the stability analysis and control synthesis on fuzzy system focus on Lyapunov asymptotic stability, which is defined over an infinite time interval. However, in some practical engineering applications, the finite time control is of practical significance. Finite time stability admits that the state does not exceed a certain bound during a fixed finite time interval, see for example, [21-29]. Among them, Shen et al. discussed the problem of finite-time reliable $L_{2}-L_{\infty} / H_{\infty}$ control for Takagi-Sugeno fuzzy systems with actuator faults without considering time-delay in [21]. In [22], finite-time $H_{\infty}$ fuzzy control of nonlinear jump systems with time delay via dynamic observer-based state feedback was studied, in which, the time-delay is constant. Non-fragile finite-time filter was designed for time-delayed Markovian jumping systems via T-S fuzzy model approach in [27]. To the best knowledge of our author, there are few articles on finite time non-fragile dissipative control for uncertain TS fuzzy system with time-varying delay.

Motivated by the above discussion, in this paper, we consider the problem of finite time non-fragile control for uncertain TS fuzzy system with time-varying delay subject to $(Q, V, R)-\alpha$-dissipative. The main contributions of this paper are summarized as follows: 1) a new integral inequality is introduced to solve the integral in the derivation of the Lyapunov function; 2) sufficient conditions were obtained to ensure that the closed-loop system is finite time bounded and dissipative; 3) the non-fragile dissipative controller can be obtained by solving the LMIs; 4) the dissipativity can be seen from Fig.4 directly; 5) the simulation results demonstrate the feasibility of the method.

Notation: Throughout this paper, $\mathrm{R}^{n}$ denotes the -dimensional Euclidean space, and $\mathrm{R}^{n \times m}$ is the set of real matrices. For $A \in \mathrm{R}^{n \times m}, A^{-1}$ and $A^{\mathrm{T}}$ denote the matrix inverse and matrix transpose respectively. $\lambda(A)$ means the eigenvalue of $A$. For a real symmetric matrix $A \in \mathrm{R}^{n \times n}, A>0(A \geq 0)$ means that $A$ is positive defined (positive semi-defined). The symbol $*$ means the symmetric term in a symmetric matrix.

\section{Problem formulation}

Consider a nonlinear system, and it can be presented as the following TS fuzzy model:

Plant rule $i$ : IF $\varepsilon_{1}(t)$ is $M_{i 1}$ and $\varepsilon_{2}(t)$ is $M_{i 2} \ldots \varepsilon_{p}(t)$ is $M_{i p}$, THEN

$$
\begin{aligned}
& \dot{x}(t)=\bar{A}_{i} x(t)+\bar{A}_{d i} x(t-d(t))+\bar{B}_{i} u(t)+\bar{B}_{\omega i} \omega(t), \\
& z(t)=\bar{C}_{i} x(t)+\bar{C}_{d i} x(t-d(t))+\bar{D}_{i} u(t)+\bar{D}_{\omega i} \omega(t), \\
& x(t)=\phi(t), t \in[-d, 0],
\end{aligned}
$$

where $i \in \Re:=\{1,2 \ldots r\}, r$ is the number of IF-THEN rules. $\varepsilon(t)=\left[\begin{array}{lllll}\varepsilon_{1}(t) & \varepsilon_{2}(t) \ldots & \varepsilon_{p}(t)\end{array}\right]^{\mathrm{T}}$ is premise variable, $M_{i k}(i=1,2 \ldots r, k=1,2 \ldots p)$ is the fuzzy set. $x(t) \in \mathrm{R}^{n}$ is the state vector, $\omega(t) \in \mathrm{R}^{q}$ is the disturbance input which belongs to $L_{2}[0, \infty) . z(t) \in \mathrm{R}^{p}$ is the control output, $\phi(t)$ is the initial condition of the system. $d(t)$ is a time-varying continuous function that satisfies $0 \leq d(t) \leq d$ and $\dot{d}(t) \leq h, h<1$. $u(t) \in \mathrm{R}^{l}$ is the control input, and for a positive scalar $b$ and time scalar $T_{c}, \int_{0}^{T_{c}} \omega^{\mathrm{T}}(t) \omega(t) \leq b . \bar{A}_{i}=$ $A_{i}+\Delta A_{i}, \bar{A}_{d i}=A_{d i}+\Delta A_{d i}, \bar{B}_{i}=B_{i}+\Delta B_{i}, \bar{B}_{\omega i}=B_{\omega i}+\Delta B_{\omega i}, \bar{C}_{i}=C_{i}+\Delta C_{i}, \bar{C}_{d i}=C_{d i}+\Delta C_{d i}, \bar{D}_{i}=$ $D_{i}+\Delta D_{i}, \bar{D}_{\omega i}=D_{\omega i}+\Delta D_{\omega i}$. And $A_{i}, A_{d i}, B_{i}, B_{\omega i}, C_{i}, C_{d i}, D_{i}, D_{\omega i}$ are known real constant matrices with appropriate dimensions; $\Delta A_{i}, \Delta A_{d i}, \Delta B_{i}, \Delta B_{\omega i}, \Delta C_{i}, \Delta C_{d i}, \Delta D_{i}, \Delta D_{\omega i}$ are unknown matrices representing norm-bounded parametric uncertainties and are assumed to be of the form:

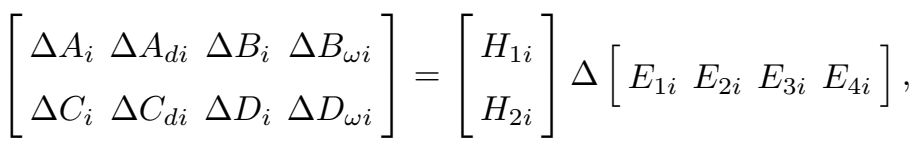

where $H_{1 i}, H_{2 i}, E_{1 i}, E_{2 i}, E_{3 i}, E_{4 i}$ are known real constant matrices with appropriate dimensions and $\Delta$ is unknown real and possibly time-varying matrices satisfying $\Delta^{\mathrm{T}} \Delta \leq I$. 
Using singleton fuzzifier, product inference, and center-average defuzzifier, the global dynamics of the TS system (1) is described by the convex sum form:

$$
\begin{aligned}
& \dot{x}(t)=\sum_{i=1}^{r} h_{i}(\varepsilon(t))\left[\bar{A}_{i} x(t)+\bar{A}_{d i} x(t-d(t))+\bar{B}_{i} u(t)+\bar{B}_{\omega i} \omega(t)\right] \\
& z(t)=\sum_{i=1}^{r} h_{i}(\varepsilon(t))\left[\bar{C}_{i} x(t)+\bar{C}_{d i} x(t-d(t))+\bar{D}_{i} u(t)+\bar{D}_{\omega i} \omega(t)\right] \\
& x(t)=\phi(t), t \in[-d, 0]
\end{aligned}
$$

where $h_{i}(\varepsilon(t))=\beta_{i}(\varepsilon(t)) / \sum_{i=1}^{r} \beta_{i}(\varepsilon(t)), \beta_{i}(\varepsilon(t))=\prod_{j=1}^{p} M_{i j}\left(\varepsilon_{j}(t)\right)$, and $M_{i j}\left(\varepsilon_{j}(t)\right)$ is the grade of membership of $\varepsilon_{j}(t)$ in $M_{i j}$. It is easy to see that $\beta_{i}(\varepsilon(t)) \geq 0$ and $\sum_{i=1}^{r} \beta_{i}(\varepsilon(t)) \geq 0$. Hence, we have $h_{i}(\varepsilon(t)) \geq 0$ and $\sum_{i=1}^{r} h_{i}(\varepsilon(t))=1$. In the sequel, for brevity we use $h_{i}$ to denote $h_{i}(\varepsilon(t))$.

Remark 1 In [10,11], the uncertain parameters in $B_{i}$ were ignored, which are unavoidable in practice. In this paper, they are considered. That is to say, this paper popularized [10] and [11].

Now consider the following global state of fuzzy controller:

$$
u(t)=\sum_{i=1}^{r} h_{i}(\varepsilon(t))\left(K_{i}+\Delta K_{i}\right) x(t)
$$

denote $\bar{K}_{i}=K_{i}+\Delta K_{i}$, with $K_{i} \in \mathrm{R}^{n \times m}, i \in \Re$ being the constant controller gains to be determined, $\Delta K_{i}$ are perturbed matrices. Two perturbation types are considered in this section.

Type 1: the multiplicative form

$$
\Delta K_{i}=H_{3 i} \Delta_{c} E_{5 i} K_{i}
$$

Type 2: the additive form

$$
\Delta K_{i}=H_{3 i} \Delta_{c} E_{5 i}
$$

where $H_{3 i}, E_{5 i}$ are known real constant matrices, $\Delta_{c}$ is an unknown matrix satisfying $\Delta_{c}^{\mathrm{T}} \Delta_{c} \leq I$.

In this paper, we consider type 1 , then type 2 can be obtained similarly.

From (3) and (4), the closed-loop system can be obtained:

$$
\begin{aligned}
& \dot{x}(t)=\sum_{i=1}^{r} \sum_{j=1}^{r} h_{i} h_{j}\left(\bar{A}_{i}+\bar{B}_{i} \bar{K}_{j}\right) x(t)+\sum_{i=1}^{r} h_{i} \bar{A}_{d i} x(t-d(t))+\sum_{i=1}^{r} h_{i} \bar{B}_{\omega i} \omega(t), \\
& z(t)=\sum_{i=1}^{r} \sum_{j=1}^{r} h_{i} h_{j}\left(\bar{C}_{i}+\bar{D}_{i} \bar{K}_{j}\right) x(t)+\sum_{i=1}^{r} h_{i} \bar{C}_{d i} x(t-d(t))+\sum_{i=1}^{r} h_{i} \bar{D}_{\omega i} \omega(t), \\
& x(t)=\phi(t), t \in[-d, 0] .
\end{aligned}
$$

Definition 1 ([30]) For some positive constants $c_{1}, b, T_{c}$ and symmetric positive matrix $R_{c}$, the closed-loop system (7) is finite time bound subject to $\left(c_{1} c_{2} b T_{c} R_{c}\right), \forall t \in\left[\begin{array}{ll}0 & T_{c}\end{array}\right]$, if there exists scalar $c_{2}>c_{1}$, such that:

$$
\sup _{-d \leq \theta \leq 0}\left\{x^{\mathrm{T}}(\theta) R_{c} x(\theta), \dot{x}^{\mathrm{T}}(\theta) R_{c} \dot{x}(\theta)\right\} \leq c_{1} \Rightarrow x^{\mathrm{T}}(t) R_{c} x(t) \leq c_{2} .
$$

Definition 2 ([16]) The non-fragile state feedback controller (4) is said to be a finite-time dissipative controller with dissipation rate $\alpha$ for system (1), if the closed-loop system (7) is finite time bounded, and for real symmetric matrices $Q, R$, and matrix $V$, with zero initial condition, the following condition is satisfied:

$$
\Gamma(t)=\langle z, Q z\rangle_{t}+2\langle z, V \omega\rangle_{t}+\langle\omega, R \omega\rangle_{t}-\alpha\langle\omega, \omega\rangle_{t} \geq 0
$$

where $\langle\Lambda, \Gamma\rangle_{t}=\int_{0}^{t} \Lambda^{\mathrm{T}} \Gamma d t$, and we assume that $Q<0, \bar{Q}=\sqrt{-Q}$. 
Lemma 1 ([31]) Let matrices $U, \eta, W$ and $M$ are real matrices of appropriate dimensions with $M$ satisfying $M=M^{\mathrm{T}}>0$, then $M+U \eta W+W^{\mathrm{T}} \eta^{\mathrm{T}} U^{\mathrm{T}}<0$, for all $\eta^{\mathrm{T}} \eta \leq I$, if and only if there exists a scalar $\varepsilon>0$, such that

$$
M+\varepsilon^{-1} U U^{\mathrm{T}}+\varepsilon W W^{\mathrm{T}}<0 .
$$

Lemma 2 For any constant matrices $N_{1}, N_{2} \in \mathrm{R}^{n \times n}, L \in \mathrm{R}^{n \times p}$, positive-definite symmetric matrix $Z \in$ $\mathrm{R}^{n \times n}$, and time-varying delay $d(t)$, then:

$$
-\int_{t-d(t)}^{t} \dot{x}^{\mathrm{T}}(s) Z x(s) d s \leq \xi^{\mathrm{T}}(t)\left\{\Pi+d(t) Y^{\mathrm{T}} Z^{-1} Y\right\} \xi(t),
$$

where

$$
\begin{gathered}
Y=\left[\begin{array}{lll}
N_{1} & N_{2} & L
\end{array}\right], \xi^{\mathrm{T}}(t)=\left[\begin{array}{ccc}
x^{\mathrm{T}}(t) & x^{\mathrm{T}}(t-d(t)) & \omega^{\mathrm{T}}(t)
\end{array}\right], \\
\Pi=\left[\begin{array}{ccc}
N_{1}^{\mathrm{T}}+N_{1} & -N_{1}^{\mathrm{T}}-N_{1} & L \\
* & -N_{2}^{\mathrm{T}}-N_{2} & -L \\
* & * & 0
\end{array}\right] .
\end{gathered}
$$

Proof.Let $C=\left[\begin{array}{cc}Z^{1 / 2} & Z^{1 / 2} Y \\ 0 & 0\end{array}\right]$, then $C^{\mathrm{T}} C=\left[\begin{array}{cc}Z & Y \\ Y^{\mathrm{T}} & Y^{\mathrm{T}} Z^{-1} Y\end{array}\right] \geq 0$. It follows that:

$$
\int_{t-d(t)}^{t}\left[\begin{array}{c}
\dot{x}(s) \\
\xi(t)
\end{array}\right]^{\mathrm{T}}\left[\begin{array}{cc}
Z & Y \\
Y^{T} & Y^{T} Z^{-1} Y
\end{array}\right]\left[\begin{array}{l}
\dot{x}(s) \\
\xi(t)
\end{array}\right] d s \geq 0
$$

Notice that, $2 \int_{t-d(t)}^{t} \xi^{\mathrm{T}}(t) Y \dot{x}(s) d s=2 \xi^{\mathrm{T}}(t) Y^{\mathrm{T}}\left[\begin{array}{lll}I & -I & 0\end{array}\right] \xi(t)$, rearranging (10) yield (9).

Remark 2 This lemma will paly a key role in the proof, which can be used to deal with the derivation of the Lyapunov function. Considering the free-weighting matrices, the conservatism can be reduced.

\section{Main results}

Theorem 1 For positive constants $c_{1}, b, T_{c}, \delta$ and positive definite matrix $R_{c}$, closed-loop system ( 7$)$ is finite time bounded subject to $\left(c_{1} c_{2} b T_{c} R_{c}\right)$, if there exists a constant $c_{2}>0$, positive definite matrices $P, Q_{1}, Q_{2}$ and any matrices $N_{1}, N_{2}, L$, with appropriate dimensions, for $i, j \in \Re$, such that:

$$
\begin{gathered}
\Omega_{i i}<0, \\
\Omega_{i j}+\Omega_{j i}<0, \\
\left(\lambda_{2}+\lambda_{3} d+\lambda_{4} \frac{d^{2}}{2}\right) c_{1}+b\left(1-e^{-\delta T_{c}}\right)<\lambda_{1} c_{2} e^{-\delta T_{c}},
\end{gathered}
$$

where, $\Pi, Y$ are defined in lemma 2, and

$$
\begin{aligned}
& \bar{P}=R_{c}^{-1 / 2} P R_{c}^{-1 / 2}, \bar{Q}_{1}=R_{c}^{-1 / 2} Q_{1} R_{c}^{-1 / 2}, \bar{Q}_{2}=R_{c}^{-1 / 2} Q_{2} R_{c}^{-1 / 2}, \\
& \Omega_{i j}=\left[\begin{array}{ccc}
\Theta_{i j}+\Pi & d \Gamma_{1 i j}^{\mathrm{T}} & d Y^{\mathrm{T}} \\
* & -Q_{2}^{-1} & 0 \\
* & * & -Q_{2}
\end{array}\right], \Theta_{i j}=\left[\begin{array}{ccc}
\varpi_{11} & P \bar{A}_{d i} & P \bar{B}_{\omega i} \\
* & -(1-h) Q_{1} & 0 \\
* & * & -\delta I
\end{array}\right] \text {, } \\
& \varpi_{11}=P\left(\bar{A}_{i}+\bar{B}_{i} \bar{K}_{j}\right)+\left(\bar{A}_{i}+\bar{B}_{i} \bar{K}_{j}\right)^{\mathrm{T}} P-\delta P+Q_{1}, \Gamma_{1 i j}=\left[\bar{A}_{i}+\bar{B}_{i} \bar{K}_{j} \bar{A}_{d i} \bar{B}_{\omega i}\right], \\
& \lambda_{1}=\lambda_{\min }(\bar{P}), \lambda_{2}=\lambda_{\max }(\bar{P}), \lambda_{3}=\lambda_{\max }\left(\bar{Q}_{1}\right), \lambda_{4}=\lambda_{\max }\left(\bar{Q}_{2}\right) \text {. }
\end{aligned}
$$


Proof. Choose an augment Lyapunov function as following:

$$
V(x(t))=x^{\mathrm{T}}(t) P x(t)+\int_{t-d(t)}^{t} e^{\delta(t-s)} x^{\mathrm{T}}(s) Q_{1} x(s) d s+\int_{-d}^{0} \int_{t+\theta}^{t} e^{\delta(t-s)} \dot{x}^{\mathrm{T}}(s) Q_{2} \dot{x}(s) d s d \theta,
$$

along the trajectories of system (7), the corresponding time derivation of (13) is given by:

$$
\begin{aligned}
\dot{V}(x(t))= & 2 x^{\mathrm{T}}(t) P \dot{x}(t)+x^{\mathrm{T}}(t) Q_{1} x(t)-(1-\dot{d}(t)) e^{\delta d(t)} x^{\mathrm{T}}(t-d(t)) Q_{1} x(t-d(t)) \\
& +d \dot{x}^{\mathrm{T}}(t) Q_{2} \dot{x}(t)-d \int_{t-d}^{t} \dot{x}^{\mathrm{T}}(s) Q_{2} \dot{x}(s) d s \\
\leq & \delta V(x(t))+2 x^{\mathrm{T}}(t) P \dot{x}(t)+x^{\mathrm{T}}(t) Q_{1} x(t)-(1-h) x^{\mathrm{T}}(t-d(t)) Q_{1} x(t-d(t)) \\
& +d \dot{x}^{\mathrm{T}}(t) Q_{2} \dot{x}(t)-d \int_{t-d}^{t} \dot{x}^{\mathrm{T}}(s) Q_{2} \dot{x}(s) d s-\delta x^{\mathrm{T}}(t) P x(t) .
\end{aligned}
$$

Then, via lemma 2,

$$
-d \int_{t-d}^{t} \dot{x}^{\mathrm{T}}(s) Q_{2} x(s) d s \leq \xi^{\mathrm{T}}(t)\left\{\Pi+d Y^{\mathrm{T}} Q_{2}^{-1} Y\right\} \xi(t),
$$

then, it can be obtained that

$$
\begin{aligned}
& \dot{V}(x(t))-\delta V(x(t))-\delta \omega^{\mathrm{T}}(t) \omega(t) \\
& =\xi^{\mathrm{T}}(t)\left[\begin{array}{l}
\sum_{j=1}^{r} h_{i}^{2}\left(\Theta_{i i}+d \Gamma_{1 i i}^{\mathrm{T}} Q_{2} \Gamma_{1 i i}+\Pi+d Y^{\mathrm{T}} Q_{2}^{-1} Y\right) \\
+\sum_{i=1}^{r} \sum_{j=1}^{r} h_{i} h_{j}\left(\Theta_{i j}+d \Gamma_{1 i j}^{\mathrm{T}} Q_{2} \Gamma_{1 i j}+\Pi+d Y^{\mathrm{T}} Q_{2}^{-1} Y\right)
\end{array}\right] \xi(t),
\end{aligned}
$$

considering (11) and Schur complement, it yields:

$$
\dot{V}(x(t))-\delta V(x(t))-\delta \omega^{\mathrm{T}}(t) \omega(t)<0,
$$

pre- and post-multiplying (14) by $e^{-\delta t}$, and integrating it from 0 to $\left(\forall t \in\left[\begin{array}{ll}0 & T_{c}\end{array}\right]\right)$, it follows that:

$$
V(x(t))<e^{\delta t}\left[V(x(0))+\int_{0}^{t} e^{-\delta s} \omega^{\mathrm{T}}(s) \omega(s) d s\right] .
$$

Among them,

$$
\begin{aligned}
V(x(0)) & =x^{\mathrm{T}}(0) P x(0)+\int_{-d(t)}^{0} e^{-\delta s} x^{\mathrm{T}}(s) Q_{1} x(s) d s+\int_{-d}^{0} \int_{\theta}^{0} e^{-\delta s} \dot{x}^{\mathrm{T}}(s) Q_{2} \dot{x}(s) d s d \theta \\
& \leq \lambda_{2} x^{\mathrm{T}}(0) R_{c} x(0)+\left(\lambda_{3} d+\lambda_{4} \frac{d^{2}}{2}\right) \sup _{-d \leq \theta \leq 0}\left\{x^{\mathrm{T}}(\theta) R_{c} x(\theta), \dot{x}^{\mathrm{T}}(\theta) R_{c} \dot{x}(\theta)\right\} \\
& \leq\left(\lambda_{2}+\lambda_{3} d+\lambda_{4} \frac{d^{2}}{2}\right) c_{1},
\end{aligned}
$$

then, $V\left(x_{t}\right) \leq e^{\delta T_{c}}\left[\left(\lambda_{2}+\lambda_{3} d+\lambda_{4} \frac{d^{2}}{2}\right) c_{1}+b\left(1-e^{-\delta T_{c}}\right)\right]$, on the other hand, $V\left(x_{t}\right) \geq \lambda_{1} x^{\mathrm{T}}(t) R_{c} x(t)$.

Then from condition (12), we have $x^{\mathrm{T}}(t) R_{c} x(t)<c_{2}$. From definition 1, the closed-loop system (7) is finite time bounded. This completes the proof.

Remark 3 In Theorem 1, sufficient conditions are obtained to guarantee that the closed-loop is finite-time bounded. Then the Theorem 2 will give finite time dissipative conditions.

Theorem 2 For positive constants $c_{1}, b, T_{c}, \delta$, positive definite matrix $R_{c}$, and symmetric matrices $Q<$ $0, R, V$, closed-loop system (7) is finite time bounded with respect to $\left(c_{1} c_{2} b T_{c} R_{c}\right)$ and satisfies (8), if there exist constants $c_{2}>0, \beta>0$, positive definite matrices $P, Q_{1}, Q_{2}$ and any matrices $N_{1}, N_{2}, L$ with appropriate dimensions, for $i, j \in \Re$, such that (12) and the following conditions hold:

$$
\begin{aligned}
& \Psi_{i i}<0, \\
& \Psi_{i j}+\Psi_{j i}<0,
\end{aligned}
$$


where

$$
\begin{gathered}
\Psi_{i j}=\left[\begin{array}{cccc}
\Lambda_{i j}+\Pi & d \Gamma_{1 i j}^{\mathrm{T}} & d Y^{\mathrm{T}} & \Gamma_{2 i j}^{\mathrm{T}} \\
* & -Q_{2}^{-1} & 0 & 0 \\
* & * & -Q_{2} & 0 \\
* & * & * & -I
\end{array}\right], \Lambda_{i j}=\left[\begin{array}{ccc}
\varpi_{11} & P \bar{A}_{d i} & P \bar{B}_{\omega i}-\bar{C}_{i}^{\mathrm{T}} V \\
* & -(1-h) Q_{1} & -\bar{C}_{d i}^{\mathrm{T}} V \\
* & * & \varpi_{33}
\end{array}\right], \\
\Gamma_{1 i j}=\left[\begin{array}{lll}
\bar{A}_{i}+\bar{B}_{i} \bar{K}_{j} & \bar{A}_{d i} & \bar{B}_{\omega i}
\end{array}\right], \Gamma_{2 i j}=\left[\begin{array}{ccc}
\bar{Q}\left(\bar{C}_{i}+\bar{D}_{i} \bar{K}_{j}\right) & \bar{Q} \bar{C}_{d i} & \bar{Q} \bar{D}_{\omega i}
\end{array}\right], \\
\varpi_{11}=P\left(\bar{A}_{i}+\bar{B}_{i} \bar{K}_{j}\right)+\left(\bar{A}_{i}+\bar{B}_{i} \bar{K}_{j}\right)^{\mathrm{T}} P-\delta P+Q_{1}, \varpi_{33}=-\bar{D}_{\omega i}^{\mathrm{T}} V-V D_{\omega i}^{\mathrm{T}}-(R-\beta I) .
\end{gathered}
$$

Proof. Select the same Lyapunov function candidate as Theorem 1 and define the following function:

$$
J=\dot{V}\left(x_{t}\right)-\delta V\left(x_{t}\right)-z^{\mathrm{T}}(t) Q z(t)-2 \omega^{\mathrm{T}}(t) V z(t)-\omega^{\mathrm{T}}(t)(R-\beta I) \omega(t) .
$$

Using Schur complement, it can be seen from (15) that:

$$
J=\xi^{\mathrm{T}}(t)\left[\begin{array}{l}
\sum_{j=1}^{r} h_{i}^{2}\left(\Lambda_{i i}+d \Gamma_{1 i i}^{\mathrm{T}} Q_{2} \Gamma_{1 i i}+\Pi+d Y^{\mathrm{T}} Q_{2}^{-1} Y+\Gamma_{2 i i}^{\mathrm{T}} \Gamma_{2 i i}\right) \\
+\sum_{i=1}^{r} \sum_{j=1}^{r} h_{i} h_{j}\left(\Lambda_{i j}+d \Gamma_{1 i j}^{\mathrm{T}} Q_{2} \Gamma_{1 i j}+\Pi+d Y^{\mathrm{T}} Q_{2}^{-1} Y+\Gamma_{2 i j}^{\mathrm{T}} \Gamma_{2 i j}\right)
\end{array}\right] \xi(t)<0,
$$

similar as the handling method in Theorem 1 and consider the zero initial condition, it is clear that,

$$
0<V\left(x_{t}\right)<e^{\delta T_{c}} \int_{0}^{T_{c}}\left[z^{\mathrm{T}}(t) Q z(t)+2 \omega^{\mathrm{T}}(t) V z(t)+\omega^{\mathrm{T}}(t)(R-\beta I) \omega(t)\right] d t .
$$

Then, we have,

$$
\beta e^{-\delta T_{c}} \int_{0}^{T_{c}} \omega^{\mathrm{T}}(t) \omega(t) d t<\int_{0}^{T_{c}}\left[z^{\mathrm{T}}(t) Q z(t)+2 \omega^{\mathrm{T}}(t) V z(t)+\omega^{\mathrm{T}}(t) R \omega(t)\right] d t,
$$

from definition 2, the closed-loop system (7) is finite time bounded and $(Q, V, R)-\alpha$-dissipative, and the dissipation factor is $\alpha=\beta e^{-\delta T_{c}}$. This completes the proof.

Remark 4 Theorem 2 gives the sufficient conditions for the finite time dissipation of the closed-loop system. However, the conditions (12) and (15) are nonlinear matrix inequalities, by using linear matrix inequalities techniques, which will be transformed into LMIs in Theorem3.

Theorem 3 For positive constants $c_{1}, c_{2}, b, T_{c}, \alpha$, positive definite matrix $R_{c}$, and symmetric matrices $Q<$ $0, R, V$, if there exist positive definite matrices $X, \tilde{Q}_{1}, \tilde{Q}_{2}$, any matrices $\tilde{N}_{1}, \tilde{N}_{2}, \tilde{L}$, with appropriate dimensions, constants $\mu$ and $\varepsilon_{i j}>0, \chi_{i j}>0$ for $i, j \in \Re$, such that:

$$
\begin{aligned}
& {\left[\begin{array}{cc}
\Phi_{i i}^{1} & \Phi_{i i}^{2} \\
* & \Phi_{i i}^{3}
\end{array}\right]<0, } \\
& {\left[\begin{array}{cc}
\Phi_{i j}^{1} & \Phi_{i j}^{2} \\
* & \Phi_{i j}^{3}
\end{array}\right]+\left[\begin{array}{cc}
\Phi_{j i}^{1} & \Phi_{j i}^{2} \\
* & \Phi_{j i}^{3}
\end{array}\right]<0, } \\
\mu R_{c}^{-1}<X< & R_{c}^{-1}, 0<\tilde{Q}_{1}<2 R_{c}^{-1}, 0<\tilde{Q}_{2}<2 R_{c}^{-1}, \Psi<0,
\end{aligned}
$$

where 


$$
\begin{aligned}
& \Phi_{i j}^{1}=\left[\begin{array}{cccccc}
\psi_{11} & \psi_{12} & \psi_{13} & \psi_{14} & \psi_{15} & d \tilde{N}_{1}^{\mathrm{T}} \\
* & \psi_{22} & \psi_{23} & X A_{d i}^{\mathrm{T}} & X C_{d i}^{\mathrm{T}} \bar{Q} & d \tilde{N}_{2}^{\mathrm{T}} \\
* & * & \psi_{33} & B_{\omega i}^{\mathrm{T}} & D_{\omega i}^{\mathrm{T}} \bar{Q} & d \tilde{L}_{1}^{\mathrm{T}} \\
* & * & * & \psi_{44} & 0 & 0 \\
* & * & * & * & -I & 0 \\
* & * & * & * & * & -\tilde{Q}_{2}
\end{array}\right], \Phi_{i j}^{2}=\left[\begin{array}{cccc}
H_{1 i} & Y_{j}^{\mathrm{T}} E_{3 i}^{\mathrm{T}}+X E_{1 i}^{\mathrm{T}} & X B_{i} H_{3 j} & Y_{j}^{\mathrm{T}} E_{5 j}^{\mathrm{T}} \\
0 & X E_{2 i}^{\mathrm{T}} & 0 & 0 \\
-V H_{2 i} & E_{4 i}^{\mathrm{T}} & D_{i} H_{3 j} & 0 \\
H_{2 i} & 0 & B_{i} H_{3 j} & 0 \\
H_{2 i} & 0 & D_{i} H_{3 j} & 0 \\
0 & 0 & 0 & 0
\end{array}\right],
\end{aligned}
$$

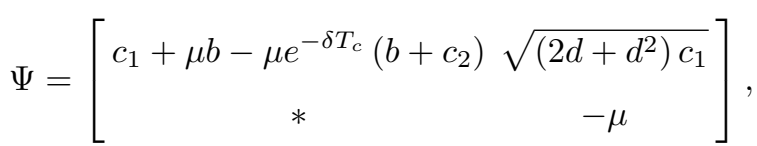

$$
\begin{aligned}
& \Phi_{i j}^{3}=\operatorname{diag}\left[-\varepsilon_{i j} I,-\chi_{i j} I,-\varepsilon_{i j} I,-\chi_{i j} I,\right], \psi_{44}=\left(-2 X+\tilde{Q}_{2}\right) / d, \\
& \psi_{11}=A_{i} X+B_{i} Y_{j}+\left(A_{i} X+B_{i} Y_{j}\right)^{\mathrm{T}}+\tilde{Q}_{1}-\delta X+\tilde{N}_{1}^{\mathrm{T}}+\tilde{N}_{1}, \psi_{12}=A_{d i} X-\tilde{N}_{1}^{\mathrm{T}}-\tilde{N}_{1}, \\
& \psi_{13}=B_{\omega i}-X C_{1 i}^{\mathrm{T}} V^{\mathrm{T}}-Y_{j}^{\mathrm{T}} D_{i}^{\mathrm{T}} V+\tilde{L}, \psi_{14}=Y_{j}^{\mathrm{T}} B_{i}^{\mathrm{T}}+X A_{i}^{\mathrm{T}}, \psi_{15}=Y_{j}^{\mathrm{T}} D_{i}^{\mathrm{T}} \bar{Q}+X C_{i}^{\mathrm{T}} \bar{Q}, \\
& \psi_{22}=-(1-h) \tilde{Q}_{1}-\tilde{N}_{2}^{\mathrm{T}}-\tilde{N}_{2}, \psi_{23}=-X C_{d 1 i}^{\mathrm{T}} V^{\mathrm{T}}-\tilde{L}, \psi_{33}=-D_{\omega i}^{\mathrm{T}} V^{\mathrm{T}}-V D_{\omega i}-(R-\beta I),
\end{aligned}
$$

then, closed-loop system (7) is finite time bounded and $(Q, V, R)-\alpha$-dissipative with dissipation factor $\alpha=\beta e^{-\delta T_{c}}$, and the controller feedback gains are given as: $K_{j}=Y_{j} X^{-1}$.

Proof.Let $X=P^{-1}$, pre- and post-multiply (16) by diag $\{X^{-1}, X^{-1}, I, I, I, X^{-1}, \underbrace{I \ldots I}_{4}\}$, and denote $Q_{1}=$ $X^{-1} \tilde{Q}_{1} X^{-1}, Q_{2}=X^{-1} \tilde{Q}_{2} X^{-1}, N_{1}=X^{-1} \tilde{N}_{1} X^{-1}, N_{2}=X^{-1} \tilde{N}_{2} X^{-1}, L=X^{-1} \tilde{L}, K_{j}=Y_{j} X^{-1}$, considering Schur complement and Lemma 1, (15) can be obtained.

On the other hand, by from $\mu R_{c}^{-1}<X<R_{c}^{-1}$, we can get $\lambda_{1}>1, \lambda_{2}<1 / \mu$, from $0<\tilde{Q}_{1}<2 R_{c}^{-1}$, namely, $0<Q_{1}<2 P R_{c}^{-1} P$, then,

$$
0<R_{c}^{-1 / 2} Q_{1} R_{c}^{-1 / 2}<2 R_{c}^{-1 / 2} P R_{c}^{-1} P R_{c}^{-1 / 2}=2 R_{c}^{-1 / 2} P R_{c}^{-1 / 2} R_{c}^{-1 / 2} P R_{c}^{-1 / 2} \leq \frac{2}{\mu^{2}} I,
$$

we can get $\lambda_{3}<2 / \mu^{2}$, and similarly, $\lambda_{4}<2 / \mu^{2}$ can be obtained from $0<\tilde{Q}_{2}<2 R_{c}^{-1}$.

Based on the above analysis, condition (12) can be guaranteed by

$$
\left(\frac{1}{\mu}+\frac{2 d}{\mu^{2}}+\frac{d^{2}}{\mu^{2}}\right) c_{1}+b\left(1-e^{-\delta T_{c}}\right)<c_{2} e^{-\delta T_{c}} .
$$

By using Schur complement, (17) can be obtained from (18), which are LMIs. This completes the proof. Remark 5 Theorem 3 gives the sufficient conditions for designing the finite-time non-fragile dissipative controller for TS fuzzy system with time-varying delay. Notice that $\alpha=\beta e^{-\delta T_{c}}$, if $\delta, T_{c}$ have been given, the value of the system dissipation rate $\alpha$ only depends on $\beta$. Therefore, we can obtain an optimal finite time non-fragile dissipative controller, then the scalar $\beta$ can reduce to the minimum possible value such that LMIs $(16,17)$ are satisfied. The optimization problem can be described as follows with $\alpha=\beta e^{-\delta T_{c}}$ :

$$
\begin{aligned}
& \min \quad c_{2}+\beta \\
& X, \tilde{Q}_{1}, \tilde{Q}_{2}, \tilde{N}_{1}, \tilde{N}_{2}, \tilde{L}, \mu, \delta \\
& \text { s.t. }(16,17) .
\end{aligned}
$$

\section{Numerical example}

Example 1 Consider the following continuous-time chaotic system with the input term in [32]: 


$$
\left\{\begin{array}{l}
\dot{x}_{1}(t)=-a x_{1}(t)+a x_{2}(t)+u(t) \\
\dot{x}_{2}(t)=c x_{1}(t)-x_{2}(t)-x_{1}(t) x_{3}(t) \\
\dot{x}_{3}(t)=x_{1}(t) x_{2}(t)-b x_{3}(t)
\end{array}\right.
$$

where $a, b, c$ are constants, $u(t)$ is the input term. Assume that $x_{1}(t) \in[-d, d] a=10, b=8 / 3, c=28$, $d=30$. When $u(t)=0$, and the initial condition is chosen as $x(0)=\left[\begin{array}{lll}1 & 0 & 0\end{array}\right]^{\mathrm{T}}$. Select the membership functions $M_{1}\left(x_{1}(t)\right)=\frac{1}{2}\left(1+\frac{x_{1}(t)}{d}\right), M_{2}\left(x_{1}(t)\right)=\frac{1}{2}\left(1-\frac{x_{1}(t)}{d}\right)$. On the basis of [32], we can use the following TS fuzzy system with two fuzzy rules to present the nonlinear equation:

Rule 1: IF $x_{1}(t)$ is $M_{1}\left(x_{1}(t)\right)$, THEN $\dot{x}(t)=A_{1} x(t)+B u(t)$,

Rule 2: IF $x_{1}(t)$ is $M_{2}\left(x_{1}(t)\right)$, THEN $\dot{x}(t)=A_{2} x(t)+B u(t)$,

where

$$
A_{1}=\left[\begin{array}{ccc}
-a & a & 0 \\
c & -1 & -d \\
0 & d & -b
\end{array}\right], A_{2}=\left[\begin{array}{ccc}
-a & a & 0 \\
c & -1 & d \\
0 & -d & -b
\end{array}\right], B=\left[\begin{array}{l}
1 \\
0 \\
0
\end{array}\right] .
$$

For given $c_{1}=1, c_{2}=2, R=I_{3}, \delta=0.001, T_{c}=10$. Firstly, on the concept of the parallel distributed compensation method (PDC), the fuzzy controller can be chosen as: $u(t)=\sum_{i=1}^{2} h_{i} K_{i} x(t)$, using the matlab toolbox, we can get the following controller gain matrices:

$$
K_{1}=\left[\begin{array}{lll}
7.3482 & -53.1680-4.7128
\end{array}\right], K_{2}=\left[\begin{array}{lll}
7.3482 & -53.1680 & 4.7128
\end{array}\right] .
$$

The response of the state of closed-loop system can be seen from Fig. 1.

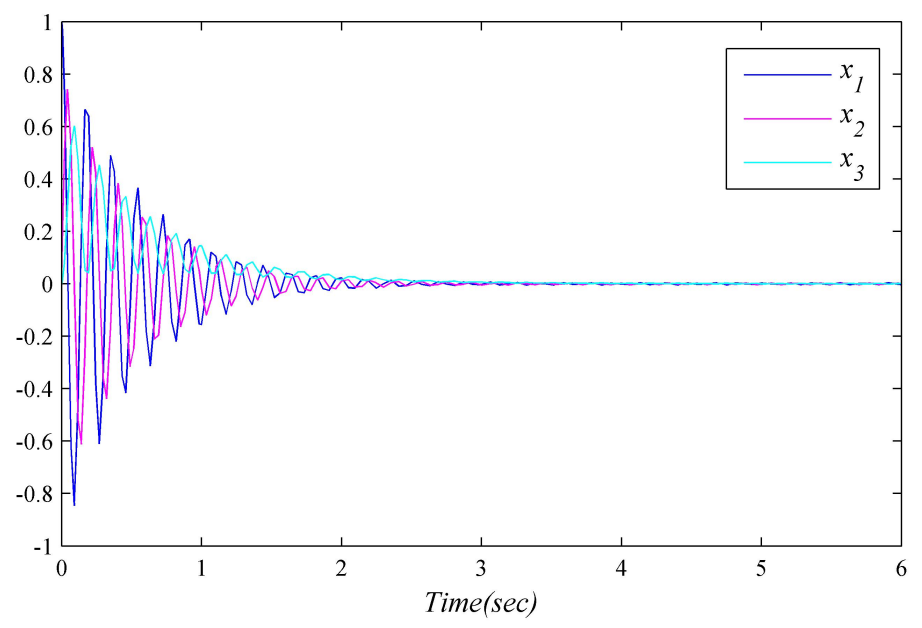

Fig. 1. State response of the closed-loop system (PDC)

Then, using the non-fragile controller (4), with perturbed matrices $H_{31}=0.1, H_{32}=0.3, E_{51}=E_{52}=$ -0.1 , we can get the matrices gains are

$$
K_{1}=\left[\begin{array}{ll}
-3.9310-39.4744-12.9593
\end{array}\right], K_{2}=\left[\begin{array}{lll}
-3.8595 & -39.4447 & 12.9211
\end{array}\right] .
$$

The response of the state of closed-loop system can be seen from Fig. 2.

Remark 6 From Fig.1 and Fig.2, it can be seen easily that the non-fragile controller in this paper can trend stable faster. So the non-fragile controller in this paper is superior then the PDC controller. 


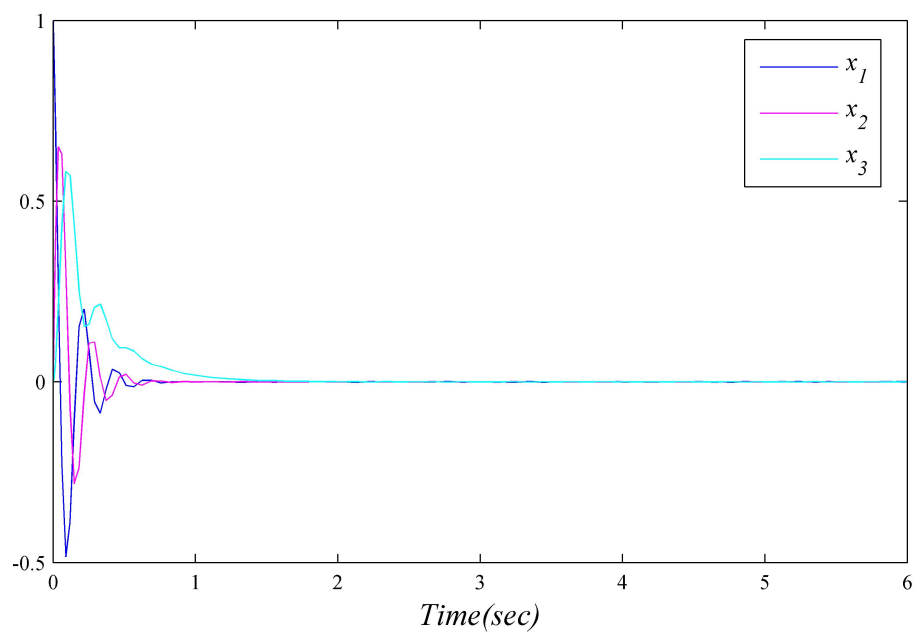

Fig. 2. State response of the closed-loop systemnon-fragile

Example 2 Consider TS fuzzy (1) with two fuzzy rules with the following parameters:

$$
\begin{aligned}
& A_{1}=\left[\begin{array}{cc}
-1 & 1 \\
1 & -2
\end{array}\right], A_{2}=\left[\begin{array}{cc}
1 & 1 \\
0.1 & 0.5
\end{array}\right], A_{d 1}=\left[\begin{array}{cc}
0.1 & 0 \\
0 & 0.1
\end{array}\right], A_{d 2}=\left[\begin{array}{cc}
1 & 0 \\
0.1 & -0.2
\end{array}\right], B_{\omega 1}=\left[\begin{array}{cc}
0.01 & 0 \\
0 & 0
\end{array}\right], \\
& B_{\omega 2}=\left[\begin{array}{cc}
0 & 0 \\
0 & 0.01
\end{array}\right], B_{1}=\left[\begin{array}{c}
0 \\
0.1
\end{array}\right], B_{2}=\left[\begin{array}{c}
1 \\
-0.1
\end{array}\right], D_{1}=\left[\begin{array}{l}
0 \\
1
\end{array}\right], D_{2}=\left[\begin{array}{c}
0 \\
0.5
\end{array}\right], C_{1}=C_{d 1}=\left[\begin{array}{cc}
1 & 0 \\
1 & 0
\end{array}\right], \\
& C_{2}=C_{d 2}=\operatorname{diag}\{0.5,0.5\}, D_{\omega 1}=D_{\omega 2}=\operatorname{diag}\{0.01,0.01\}, H_{i j}=\left[\begin{array}{ll}
-0.03 & 0.03
\end{array}\right]^{\mathrm{T}}(i, j=1,2) \text {, } \\
& E_{21}=E_{22}=[-0.35-0.45], E_{31}=E_{32}=-0.15, E_{41}=E_{42}=[-0.5-0.4], \\
& H_{31}=0.1, H_{32}=0.3, E_{51}=E_{52}=-0.1, Q=\operatorname{diag}\{-0.01,-0.01\}, V=\operatorname{diag}\{0.1,0.1\} \text {, } \\
& R=\operatorname{diag}\{5,8\}, c_{1}=1, c_{2}=10, T_{c}=3, b=0.5, h=0, d=1.027, R_{c}=I_{2}, \delta=0 .
\end{aligned}
$$

Select membership function are $h_{1}(t)=1 /\left[1+\exp \left(0.5 x_{1}(t+1)\right)\right], h_{2}(t)=1-h_{1}(t) \omega(t)=\exp (-t) \sin (t)$. Using the matlab toolbox solving the LMIs, we can obtain the matrices gains are:

$$
K_{1}=[-3.2579-0.7768], K_{2}=[-1.8009-1.7832] .
$$

Then, the response of the closed-loop system can be seen from Fig.3. Finally let the dissipation factor $\alpha=1$, Fig 4. Plot the simulation of $\Gamma(t)$.

Remark 7 It can be seen from Fig.3, the closed-loop system is finite time bounded subject to the non-fragile controller. From Fig. 4, we can see (8) is satisfied, which means the closed-loop system is dissipative from Definition 2.

\section{Conclusion}

The problem of finite time non-fragile dissipative control for uncertain TS fuzzy time-varying delay system has been considered in this paper. Sufficient delay-dependent conditions have been obtained which guarantee that the closed-loop system is not only dissipative, but also finite-time bounded for all uncertainties. Based on the derived conditions, the non-fragile dissipative controller can be obtained by solving the LMIs. The results in this paper are less conservative. Finally, simulation results demonstrate the feasibility of the proposed method.

Acknowledgment This Letter was supported by the National Natural Science Foundation of China No. 61273004, and the Natural Science Foundation of Hebei province No. F2014203085. 


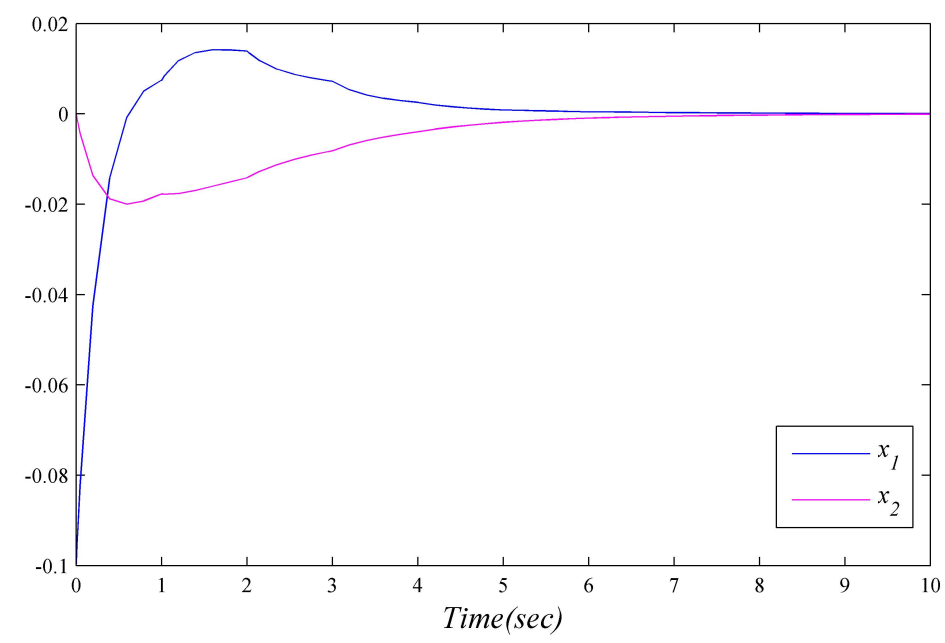

Fig. 3. State response of the closed-loop system

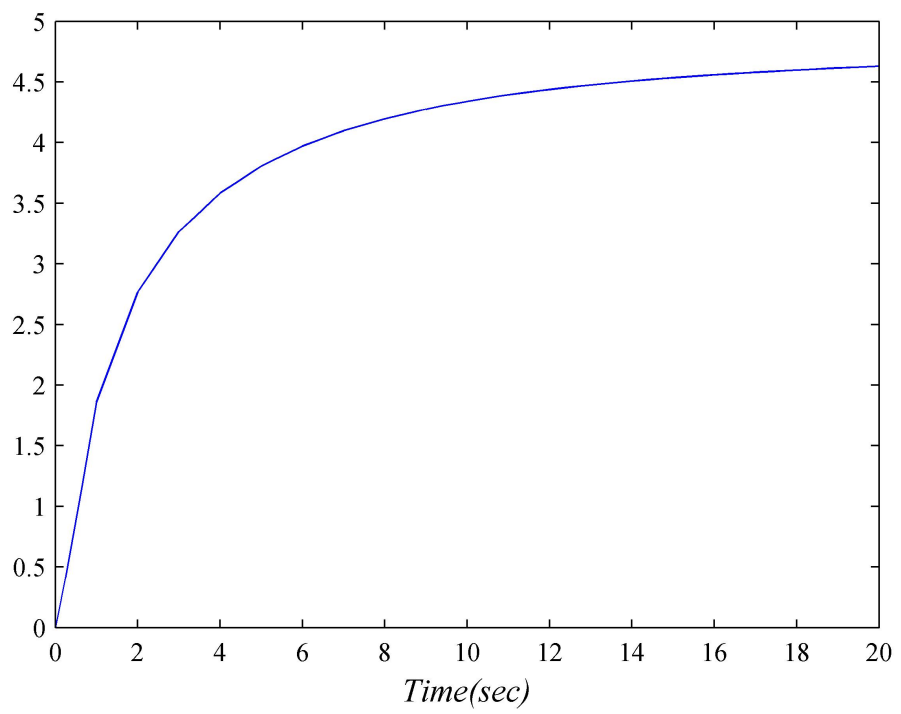

Fig. 4. The simulation of $\Gamma(t)$

\section{References}

[1] H. Shen, Z. Wang, X. Huang, J. Wang, Fuzzy dissipative control for nonlinear Markovian jump systems via retarded feedback, Journal of the Franklin Institute, 351(7) (2014) 3797-3817.

[2] Z. Y. Zhang, C. Lin, B. Chen, New stability and stabilization conditions for T-S fuzzy systems with time delay, Fuzzy Sets and Systems, 263 (2015) 82-91.

[3] L. Su, H. Shen, Fault-tolerant dissipative synchronization for chaotic systems based on fuzzy mixed delayed feedback, Neurocomputing, 151 (2015) 1407-1413.

[4] H. Y. Li, S. Yin, Y. N. Pan, H. K. Lam, Model reduction for interval type-2 Takagi-Sugeno fuzzy systems, Automatica, 61 (2015) 308-314.

[5] H. Y. Li, Y. N. Pan, Q. Zhou, Filter design for interval type-2 fuzzy systems with D stability constraints under a unified frame, IEEE Transactions on Fuzzy Systems, 23 (2015) 719-725. 
[6] Y. C. Ma, M. H. Chen, Q.L. Zhang, Memory dissipative control for singular T-S fuzzy time-varying delay systems under actuator saturation. Journal of the Franklin institutes, 352 (2015) 3947-3970.

[7] H. C. Yan, T. T. Wang, H. Zhang, H. B. Shi, Event-triggered $H_{\infty}$ control for uncertain networked T-S fuzzy systems with time delay Neurocomputing, 157 (2015) 273-279.

[8] Y. S. Zhang, S. Y. Xu, Y. Zou, J. Y. Lu, Delay-dependent robust stabilization for uncertain discrete-time fuzzy Markovian jump systems with mode-dependent time delays, Fuzzy Sets and Systems, 164 (1) (2011) 66-81.

[9] J. H. Zhang, P. Shi, H. J. Yang, Non-fragile robust stabilization and $H_{\infty}$ control for uncertain stochastic nonlinear timedelay systems, Chaos Solitons Fractals, 42 (5) (2009) 3187-3196.

[10] Y. C. Ma, N. N. Gu, Q.L. Zhang, Non-fragile robust $H_{\infty}$ control for uncertain discrete-time singular systems with timevarying delays, Journal of the Franklin Institute, 351 (6) (2014) 3163-3181.

[11] S. P. He, Non-fragile passive controller design for nonlinear Markovian jumping systems via observer-based controls, Neurocomputing, 147 (2015) 350-357.

[12] B. Y. Zhang, S. S. Zhou, T. Li, A new approach to robust and non-fragile $H_{\infty}$ control for uncertain fuzzy systems, Information Science, 177 (2007) 5118-5133.

[13] C. Wang, Y. Shen, Delay-dependent non-fragile robust stabilization and $H_{\infty}$ control of uncertain stochastic systems with time-varying delay and nonlinearity Journal of the Franklin Institute, 348 (2011) 2174-2190.

[14] M. Kchaou, A. ElHajjaji, A. Toumi, Non-fragile $H_{\infty}$ output feedback control design for continuous-time fuzzy systems, ISA Transactions, 54 (2015) 3-14.

[15] J. C. Willems, Dissipative dynamical systems, part I: general theory, Archive for Rational Mechanics and Analysis, 45(5) (1972) 321-351.

[16] H. Gassara, A.El Hajjaji, M. Kchaou, M. Chaabane, Observer based $(Q, V, R)-\alpha$-dissipative control for T-S fuzzy descriptor systems with time delay, Journal of the Franklin Institute, 351 (2014) 187-206.

[17] Z. T. Gong, Y. B. Shao, Global existence and uniqueness of solutions for fuzzy differential equations under dissipative-type conditions, Computers and Mathematics with Applications, 56 (2008) 2716-2723.

[18] V. Lupulescu, Initial value problem for fuzzy differential equations under dissipative conditions, Information Sciences, 178 (2008) 4523-4533.

[19] S. Muralisankar, N. Gopalakrishnan, P. Balasubramaniam, An LMI approach for global robust dissipativity analysis of T-S fuzzy neural networks with interval time-varying delays, Expert Systems with Applications, 39 (2012) $3345-3355$.

[20] H. Shen, Z. Wang, X. Huang, J. Wang, Fuzzy dissipative control for nonlinear Markovian jump systems via retarded feedback, Journal of the Franklin Institute, 351 (7) (2014) 3797-3817.

[21] H. Shen, J. H. Park, Z. G. Wu, Finite-time reliable $L_{2}-L_{\infty} / H_{\infty}$ control for Takagi-Sugeno fuzzy systems with actuator faults, IET control theory and Applications, 8(9) (2014) 688-696.

[22] S. P. He, F. Liu, Finite-time $H_{\infty}$ fuzzy control of nonlinear jump systems with time delay via dynamic observer-based state feedback, IEEE Transactions on fuzzy systems, 20(4) (2012) 605-614.

[23] Y. Q. Zhang, P. Shi, S. K. Nguangd, H. R. Karimi, R. K. Agarwal, Robust finite-time fuzzy $H_{\infty}$ control for uncertain time-delay systems with stochastic jumps, Journal of the Franklin Institute, 351 (2014) 4211-4229.

[24] J. Cheng, H. Zhu, S. M. Zhong, Y. Zeng, X. C. Dong, Finite-time $H_{\infty}$ control for a class of Markovian jump systems with mode-dependent time-varying delays via new Lyapunov functional, ISA Transactions, 52 (2013) 768-774.

[25] Y. C. Ma, L. Fu, Y. H. Jing, Q. L. Zhang, Finite-time $H_{\infty}$ control for a class of discrete-time switched singular time-delay systems subject to actuator saturation, Applied Mathematics and Computation, 261 (2015) $264-283$.

[26] H. Liu, G. P. Zhou, Finite-time sampled-data control for switching T-S fuzzy systems, Neurocomputing, 166 (2015) 294-300.

[27] S. P. He, H. L. Xu, Non-fragile finite-time filter design for time-delayed Markovian jumping systems via T-S fuzzy model approach Nonlinear Dynamics, 80 (2015) 1159-1171.

[28] M. J. Cai, Z. R. Xiang, Adaptive fuzzy finite-time control for a class of switched nonlinear systems with unknown control coefficients, Neurocomputing, 162 (2015) 105-115.

[29] S. P. He, Energy-to-peak filtering for T-S fuzzy systems with Markovian jumping: The finite-time case, Neurocomputing, 168 (2015) 348-355.

[30] F. Amato, M. Ariola,, C. Cosentino, Finite time stability of linear time-varying systems analysis and controller design, IEEE Transactions on Automatic Control, 55 (4) (2010) 1003-1008.

[31] I. R. Petersen, C. V. Hollot, A Riccati equation approach to the stabilization of uncertain linear systems, Automatica, 22 (4) (1986) 397-411.

[32] K. Tanaka, T. Ikeda, H. O. Wang, A unified approach to controlling chaos via an LMI-based fuzzy control system design, IEEE Transactions on circuits and systems I:Fundamental Theory and Applications, 45(10) (1998) 1021-1040. 